\title{
Our experience in second line Anti Retroviral Therapy (ART) At State Aids Clinical Expert Panel (SACEP) Clinic, Centre of Excellence (CoE), Art Centre, B. J. Medical College, Civil Hospital, Ahmedabad
}

Bankim Mankad, Hemang Purohit, Asha Shah, Manoj Shevkani", Burzin Kavina, Bipin Amin, Umesh Nihalani, Sanjeev Prajapati, Urvi Derasari, Girish Prajapati

From $16^{\text {th }}$ International Symposium on HIV and Emerging Infectious Diseases Marseille, France. 24-26 March 2010

\section{Background}

To share our experience in second line Anti Retroviral Therapy (ART) at State AIDS Clinical Expert Panel (SACEP) Clinic, Centre of Excellence (CoE), ART Centre, B. J. Medical College, Civil Hospital, Ahmedabad, Gujarat, India.

\section{Methods}

First line treatment failure Patients (as per Indian National ART Guidelines) were referred to SACEP Clinic for further evaluation and if eligible as per Indian National $2^{\text {nd }}$ Line ART Guidelines; enrolled and followed up for initiation of $2^{\text {nd }}$ Line ART at our institute.

Table 1

\begin{tabular}{|c|c|c|c|}
\hline \multicolumn{3}{|c|}{ Parameter } & Outcome \\
\hline \multicolumn{3}{|c|}{ Total Patients referred to SACEP } & 127 \\
\hline \multicolumn{3}{|c|}{ Patients Eligible and referred for Plasma Viral Load (PVL) $(n=127)$} & $122(96.06 \%)$ \\
\hline \multicolumn{3}{|c|}{ Patients recommended $2^{\text {nd }}$ Line ART after PVL $(n=122)$} & $75(61.47 \%)$ \\
\hline \multicolumn{3}{|c|}{ Patients initiated Free $2^{\text {nd }}$ Line ART $(n=75)$} & $69(92.0 \%)$ \\
\hline \multicolumn{3}{|c|}{ Patients under follow up TB Treatment/Counseling to initiate $2^{\text {nd }}$ Line ART } & 6 \\
\hline \multicolumn{3}{|c|}{ Adherence Counseling for $2^{\text {nd }}$ Line ART Patients $(n=69)$} & $69(100 \%)$ \\
\hline \multicolumn{3}{|c|}{ Undetectable PVL $(<400$ copies $/ \mathrm{ml})$ after 6 months of $2^{\text {nd }}$ Line ART $(n=28)$} & 24 \\
\hline \multicolumn{3}{|c|}{ Therapeutic Success according to Undetectable PVL (24/28) } & $85.74 \%$ \\
\hline \multicolumn{3}{|c|}{ No. of Death after initiation of $2^{\text {nd }}$ Line ART $(n=69)$} & $8(11.59 \%)$ \\
\hline \multirow[t]{9}{*}{ Death Analysis } & Cause of Death & WHO Stage/PVL/CD4 & $n=8$ \\
\hline & Accidental & T3/560459/17 & 1 \\
\hline & Renal Failure & T3/208048/230 & 1 \\
\hline & Malignancy & $\mathrm{T} 4 / 384469 / 38$ & 1 \\
\hline & \multirow[t]{4}{*}{ Opportunistic Infections (Ols) } & T3/46453/67 & 4 \\
\hline & & $\mathrm{T} 4 / 507495 / 26$ & \\
\hline & & $\mathrm{T} 4 / 1166325 / 32$ & \\
\hline & & $\mathrm{T} 4 / 289375 / 21$ & \\
\hline & Unknown Reason & T3/4095718/29 & 1 \\
\hline
\end{tabular}

\footnotetext{
* Correspondence: coe.art.ahmedabad@gmail.com
}

ART center B J Medical College, Ahmedabad, India 


\section{Results}

Provision of Free $2^{\text {nd }}$ Line ART to People Living with HIV/AIDS (PLHA) Program by National AIDS Control Organization (NACO), India was started in November, 2008 under SACEP Clinic for Gujarat and Rajasthan PLHAs. Till October 2009 total $1271^{\text {st }}$ Line ART Failure patients were referred to SACEP Clinic, Table 1.

\section{Discussion}

Switching to $2^{\text {nd }}$ Line ART based on Immunological Failure, Clinical Failure is not recommended; as out of 122 PLHAs analyzed only 75 PLHAs have Virological Failure. Adherence Counseling for $2^{\text {nd }}$ Line ART helps quite in reduction of PVL. Deaths analysis shows that late switch to $2^{\text {nd }}$ Line ART at CD $4<100$ cells $/ \mathrm{mm}^{3}$ may not result in desired therapeutic goals.

Published: 11 May 2010

doi:10.1186/1742-4690-7-S1-P53

Cite this article as: Mankad et al:: Our experience in second line Anti

Retroviral Therapy (ART) At State Aids Clinical Expert Panel (SACEP)

Clinic, Centre of Excellence (CoE), Art Centre, B. J. Medical College, Civil

Hospital, Ahmedabad. Retrovirology 2010 7(Suppl 1):P53.

Submit your next manuscript to BioMed Central and take full advantage of:

- Convenient online submission

- Thorough peer review

- No space constraints or color figure charges

- Immediate publication on acceptance

- Inclusion in PubMed, CAS, Scopus and Google Scholar

- Research which is freely available for redistribution

Submit your manuscript at www.biomedcentral.com/submit 